\title{
Beating of oscillations in transport coefficients of a one-dimensionally periodically modulated two-dimensional electron gas in the presence of spin-orbit interaction
}

\author{
X. F. Wang* and P. Vasilopoulos ${ }^{\dagger}$ \\ Department of Physics, Concordia University, 1455 de Maisonneuve Ouest, Montréal, Québec, Canada H3G 1 M8 \\ F. M. Peeters \\ Departement Fysica, Universiteit Antwerpen (Campus Drie Eiken), Universiteitsplein 1, B-2610 Antwerpen, Belgium
}

(Received 26 August 2004; revised manuscript received 8 November 2004; published 1 March 2005)

\begin{abstract}
Transport properties of a two-dimensional electron gas (2DEG) are studied in the presence of a perpendicular magnetic field $B$, of a weak one-dimensional (1D) periodic potential modulation, and of the spin-orbit interaction (SOI) described only by the Rashba term. In the absence of the modulation the SOI mixes the spin-up and spin-down states of neighboring Landau levels into two new, unequally spaced energy branches. The levels of these branches broaden into bands in the presence of the modulation and their bandwidths oscillate with the field $B$. Evaluated at the Fermi energy, the $n$th level bandwidth of each series has a minimum or vanishes at different values of the field $B$. In contrast with the 1D-modulated 2DEG without SOI, for which only one flat-band condition applies, here there are two flat-band conditions that can change considerably as a function of the SOI strength $\alpha$ and accordingly influence the transport coefficients of the 2DEG. The phase and amplitude of the Weiss and Shubnikov-de Haas $(\mathrm{SdH})$ oscillations depend on the strength $\alpha$. For small values of $\alpha$ both oscillations show beating patterns. Those of the former are due to the independently oscillating bandwidths whereas those of the latter are due to modifications of the density of states, exhibit an even-odd filling factor transition, and are nearly independent of the modulation strength. For strong values of $\alpha$ the $\mathrm{SdH}$ oscillations are split in two.
\end{abstract}

DOI: $10.1103 /$ PhysRevB.71.125301

PACS number(s): 73.20.-r, 73.43.Qt, 73.61.-r, 85.75.-d

\section{INTRODUCTION}

The magnetotransport of the two-dimensional electron gas (2DEG), subjected to periodic potential modulations, has attracted considerable experimental ${ }^{1}$ and theoretical ${ }^{2,3}$ attention during the last two decades. For one-dimensional (1D) modulations novel oscillations of the magnetoresistivity tensor $\rho_{\mu \nu}$ have been observed, at low magnetic fields $B$, distinctly different in period and temperature dependence from the usual Shubnikov-de Haas $(\mathrm{SdH})$ ones observed at higher $B$. These novel oscillations, referred to as the Weiss oscillations, reflect the commensurability between two length scales: the cyclotron diameter at the Fermi level $2 R_{c}$ $=2 \sqrt{2 \pi n_{e}} \ell_{c}^{2}$, with $n_{e}$ the electron density and $\ell_{c}$ the magnetic length, and $a$ the period of the potential modulation.

The emerging field of spintronics brought into the fore the importance of spin-orbit interaction (SOI) in a variety of situations. It is important in the development of spin-based transistors, ${ }^{4}$ possibly in future quantum computations, ${ }^{5}$ in an unexpected metal-to-insulator transition in two-dimensional (2D) - Ref. 6-hole gas, in spin-resolved ballistic transport, ${ }^{7}$ in Aharonov-Casher experiments, ${ }^{8}$ in spin-galvanic ${ }^{9}$ and spin valve $^{10}$ effects, in the spin-Hall effect, ${ }^{11}$ etc. The effect is important in inversely asymmetric bulk semiconductor crystals, due to the internal crystal field, as well in asymmetrically confined semiconductor heterostructures. In the former case the contributions to the spin splitting in the conduction band vary as a $\sim k^{3}$ term and dominate in wide-gap structures $^{12}$ whereas in the latter vary as a $\sim k$ term, referred to as the Rashba term, and dominate in narrow-gap structures. ${ }^{13}$ The latter was confirmed by experiments that showed a zero-magnetic-field spin splitting for carriers with finite momentum in a modulation-doped GaAs/AlGaAs heterojunction ${ }^{14}$ as well as by magnetotransport measurements in a $2 \mathrm{D}$ hole system. ${ }^{15}$ The explanation proposed by Bychkov and Rashba ${ }^{16}$ employed the Rashba spin-orbit Hamiltonian, in which the spin of finite-momentum electrons feels a magnetic field perpendicular to the electron momentum in the inversion plane. A detailed account of magnetotransport of the $2 \mathrm{DEG}$ in the presence of SOI but absence of modulations appeared recently. ${ }^{17}$

Given the importance the SOI has acquired, one question that arises concerns its influence on magnetotransport properties of a 2DEG in the presence of periodic potential modulations. So far we are aware of only the brief, classical study of Ref. 18. Since some effects of the modulations can be explained only quantum mechanically, ${ }^{3}$ it is of interest to reexamine the problem quantum mechanically. This is the subject of this paper. We will consider only weak 1D modulations and make use of our experience with the ${ }^{3}$ and with the unmodulated 2DEG in the presence of SOI. ${ }^{17}$ The main qualitative findings are as follows. The levels of the + and - , unequally spaced energy branches, due to the SOI when the modulation is absent, broaden into bands when the modulation is present and their bandwidths oscillate with the field $B$. Evaluated at the Fermi energy, these bandwidths vanish at different values of the field $B$ and modify considerably the flat-band condition and the transport coefficients as a function of the SOI strength $\alpha$. As a result, the phase and amplitude of the commensurability and $\mathrm{SdH}$ oscillations change when $\alpha$ is varied. For small values of $\alpha$ the former show a beating pattern while for strong values of $\alpha$ the latter are split in two. 
In the next section we present the one-electron eigenfunctions and eigenvalues. Analytical results for the conductivities are given in Sec. III and numerical results in Sec. IV. The last section contains a summary and concluding remarks.

\section{EIGENFUNCTIONS AND EIGENVALUES}

\section{A. A 2DEG in the presence of SOI and absence of potential modulation}

We consider a 2DEG in the $(x-y)$ plane and a magnetic field along the $z$ direction. In the Landau gauge $\mathbf{A}$ $=(0, B x, 0)$ the one-electron Hamiltonian including the Rashba term reads

$$
H_{0}=\frac{(\mathbf{p}+e \mathbf{A})^{2}}{2 m^{*}}+\frac{\alpha}{\hbar}[\boldsymbol{\sigma} \times(\mathbf{p}+e \mathbf{A})]_{z}+\frac{1}{2} g \mu_{B} B \sigma_{z},
$$

where $\mathbf{p}$ is the momentum operator of the electrons, $m^{*}$ is the effective electron mass, $g$ the Zeeman factor, $\mu_{B}$ the Bohr magneton, $\boldsymbol{\sigma}=\left(\sigma_{x}, \sigma_{y}, \sigma_{z}\right)$ the Pauli spin matrices, and $\alpha$ the strength of the SOI or Rashba parameter.

Using the Landau wave functions without SOI as a basis, we can express the new eigenfunction in the form:

$$
\Psi_{k_{y}}(\mathbf{r})=e^{i k_{y} y} \sum_{n=0}^{\infty} \phi_{n}\left(x+x_{c}\right)\left(\begin{array}{c}
C_{n}^{+} \\
C_{n}^{-}
\end{array}\right) / \sqrt{L_{y}} .
$$

Here $\phi_{n}(x)=e^{-x^{2} / 2 l_{c}^{2}} H_{n}\left(x / l_{c}\right) /\left(\sqrt{\pi} 2^{n} n ! l_{c}\right)^{1 / 2}$ is the harmonic oscillator function, $\omega_{c}=e \mathrm{e} / \mathrm{m}^{*}$ the cyclotron frequency, $l_{c}$ $=\left(\hbar / m^{*} \omega_{c}\right)^{1 / 2}$ the magnetic length, and the cyclotron orbit is centered at $x_{c}=l_{c}^{2} k_{y}, n$ the Landau-level index, and $|\sigma\rangle$ the electron spin written as the row vector $\langle\sigma|=(1,0)$ if it is pointing up and $(0,1)$ if it is pointing down.

Using these wave functions and Eq. (1) the eigenvalue problem $H_{0} \Psi=E \Psi$ leads to an infinite system of equations that can be solved exactly after decomposing it into independent systems of one or two equations. ${ }^{17}$ The resulting eigenstates are labeled by a new quantum number $s$ for the energy instead of $n$. For $s=0$ there is one level, the same as the lowest Landau level without SOI, with energy

$$
E_{0}^{+}=E_{0}=\hbar \omega_{c} / 2-g \mu_{B} B / 2
$$

and wave function

$$
\Psi_{0}^{+}\left(k_{y}\right)=e^{i k_{y} y} \phi_{0}\left(x+x_{c}\right)\left(\begin{array}{l}
0 \\
1
\end{array}\right) / \sqrt{L_{y}} .
$$

For $s=1,2,3, \ldots$, there are two branches of levels, denoted by + and - , with energies

$$
E_{s}^{ \pm}=s \hbar \omega_{c} \pm\left[E_{0}^{2}+2 s \alpha^{2} / l_{c}^{2}\right]^{1 / 2} .
$$

The + branch is described by the wave function

$$
\Psi_{s}^{+}\left(k_{y}\right)=\frac{e^{i k_{y} y}}{\sqrt{L_{y} \mathcal{A}_{s}}}\left(\begin{array}{r}
\mathcal{D}_{s} \phi_{s-1}\left(x+x_{c}\right) \\
\phi_{s}\left(x+x_{c}\right)
\end{array}\right)
$$

and the - one by

$$
\Psi_{s}^{-}\left(k_{y}\right)=\frac{e^{i k_{y} y}}{\sqrt{L_{y} \mathcal{A}_{s}}}\left(\begin{array}{r}
\phi_{s-1}\left(x+x_{c}\right) \\
-\mathcal{D}_{s} \phi_{s}\left(x+x_{c}\right)
\end{array}\right),
$$

where $\mathcal{A}_{s}=1+\mathcal{D}_{s}^{2}$ and $\mathcal{D}_{s}=\left(\sqrt{2 s} \alpha / l_{c}\right) /\left[E_{0}+\sqrt{E_{0}^{2}+2 s \alpha^{2} / l_{c}^{2}}\right]$.

\section{B. A 2DEG in the presence of SOI and of a 1D potential modulation}

In the presence of a $1 \mathrm{D}$ periodic electric modulation, we consider the Hamiltonian

$$
H=H_{0}+V_{0} \cos (K x),
$$

with $K=2 \pi / a$ and $a$ the modulation period. For weak modulations the energy correction due to the term $V_{0} \cos (K x)$ is evaluated by first-order perturbation theory. The results for the two branches are

$$
\begin{aligned}
E_{s}^{+}= & s \hbar \omega_{c}+\left[E_{0}^{2}+2 s \alpha^{2} / l_{c}^{2}\right]^{1 / 2}+V_{0} e^{-u / 2} \cos \left(K x_{c}\right)\left[\mathcal{D}_{s}^{2} L_{s-1}(u)\right. \\
& \left.+L_{s}(u)\right] / \mathcal{A}_{s}, \quad s=0,1, \ldots, \\
E_{s}^{-}= & s \hbar \omega_{c}-\left[E_{0}^{2}+2 s \alpha^{2} / l_{c}^{2}\right]^{1 / 2}+V_{0} e^{-u / 2} \cos \left(K x_{c}\right)\left[L_{s-1}(u)\right. \\
& \left.+\mathcal{D}_{s}^{2} L_{s}(u)\right] / \mathcal{A}_{s}, \quad s=1,2, \ldots,
\end{aligned}
$$

where $u=2 \pi^{2} l_{c}^{2} / a^{2}=K^{2} l_{c}^{2} / 2$ and $x_{c}=k_{y} l_{c}^{2} . L_{s}(u)$ is the Laguerre polynomial and for $s=0$ Eq. (9) reduces to Eq. (3) as modified by the perturbation correction. The width of the broadened levels of the two branches is given by twice the absolute value of the last term in Eqs. (9) and (10) without the $\cos \left(K \ell_{c}^{2} k_{y}\right)$ factor and is denoted by $2\left|\Delta_{s}^{ \pm}\right| . \Delta_{s}^{ \pm}$can be written in the compact form

$$
\Delta_{s}^{ \pm}=V_{0} e^{-u / 2}\left[L_{s-1 / 2 \pm 1 / 2}+\mathcal{D}_{s}^{2} L_{s-1 / 2 \mp 1 / 2}\right] / \mathcal{A}_{s},
$$

with the upper signs pertaining to the + branch and the lower ones to the - branch; obviously $\Delta_{s}^{ \pm}$is not the same for the two branches. In contrast, without SOI we have only a single branch and a single bandwidth ${ }^{3}$ and the eigenvalues are given, when the Zeeman term is neglected, by

$$
E_{n}=(n+1 / 2) \hbar \omega_{c}+V_{0} e^{-u / 2} \cos \left(K x_{c}\right) L_{n}(u)
$$

with $n$ the Landau-level index. This has consequences that will be detailed below.

As in the absence of SOI, the presence of the modulation broadens the discrete levels into bands. An important difference with the situation in which the modulation is absent is that the diagonal matrix elements of the velocity operator now do not vanish. Using $v_{y}^{ \pm}=(1 / \hbar) \partial E_{s}^{ \pm}\left(k_{y}\right) / \partial k_{y}$ their values are

$$
v_{y}^{+}=-2 V_{0} u e^{-u / 2}\left[\mathcal{D}_{s}^{2} L_{s-1}(u)+L_{s}(u)\right] \sin \left(K x_{c}\right) /\left(\hbar K \mathcal{A}_{s}\right),
$$

$$
v_{y}^{-}=-2 V_{0} u e^{-u / 2}\left[L_{s-1}(u)+\mathcal{D}_{s}^{2} L_{s}(u)\right] \sin \left(K x_{c}\right) /\left(\hbar K \mathcal{A}_{s}\right) .
$$

These nonvanishing values lead to a nonvanishing diffusive conductivity whereas in the absence of the modulation this conductivity vanishes whether the SOI is present or not. ${ }^{17}$ 
Compared to the case without SOI, we have two contributions, one from Eq. (9) and one from Eq. (10), while for $\alpha$ $=0$ we have only one value given by

$$
v_{y}=-\left(2 V_{0} / \hbar K\right) u e^{-u / 2} L_{n}(u) \sin \left(K x_{c}\right) .
$$

As a function of the magnetic field $B$, these $v_{y}^{ \pm}$contributions do not oscillate in phase due to the different dependence of the Laguerre polynomials on $B$. This modifies mostly the diffusive conductivity in the presence of the modulation and will be detailed in the following sections.

Using the asymptotic expression of the Laguerre polynomials for large $s$, we obtain $\Delta_{s}^{ \pm} \propto \cos (2 \sqrt{s u}-\pi / 4)$. The Landau level indices $s^{+}$and $s^{-}$of the corresponding branches at the Fermi energy can be determined by the equations $E_{s^{+}}^{+}$ $\approx E_{s^{-}}^{-}$and $n_{e}=\left(s^{+}+s^{-}+1\right) /\left(2 \pi l_{c}^{2}\right)$, where $n_{e}$ is the electron density. Then from the argument of $\cos (2 \sqrt{s u}-\pi / 4)$ we obtain the flat-band conditions

$$
\sqrt{u}\left[\sqrt{\pi n_{e}} l_{c} \mp \alpha /\left(\sqrt{2} \hbar \omega_{c} l_{c}\right)\right]=\pi(i-1 / 4) / 2
$$

with the upper (lower) sign corresponding to the $+(-)$ branch. Since the cyclotron radius at the Fermi energy is $R_{c}^{ \pm}=l_{c} \sqrt{2 s^{ \pm}+1}$, Eq. (16) can be written as $2 R_{c}^{ \pm} / a=i-1 / 4$ with $R_{c}^{ \pm}=R_{c}^{0} \mp \alpha / \hbar \omega_{c}$ and $R_{c}^{0}$ the cyclotron radius without SOI or $K\left(k_{F} \stackrel{\overline{+}}{+} k_{\alpha}\right) l_{c}^{2}=\pi(i-1 / 4)$ with $k_{F}=\sqrt{2 \pi n_{e}}$, and $k_{\alpha}=\alpha m^{*} / \hbar^{2}$. The same result has been obtained in Ref. 18 by a purely classical treatment. The fact that now we have two flat-band conditions, as opposed to one for $\alpha=0$, leads to oscillations with two different frequencies and consequently to beating patterns that will be shown in Sec. IV. Explicitly, writing Eq. (16) again for $i \rightarrow i+1$ and subtracting the result from Eq. (16), gives the periods in the \pm branches as $\Omega^{+}$ $=e a /\left[2 \hbar\left(k_{F}-k_{\alpha}\right)\right]$ and $\Omega^{-}=e a /\left[2 \hbar\left(k_{F}+k_{\alpha}\right)\right]$.

\section{CONDUCTIVITIES}

For weak electric fields $E_{v}$, i.e., for linear responses, and weak scattering potentials the expressions for the dc conductivity tensor $\sigma_{\mu \nu}$, in the one-electron approximation, reviewed in Ref. 19, reads $\sigma_{\mu \nu}=\sigma_{\mu \nu}^{d}+\sigma_{\mu \nu}^{\text {nd }}$ with $\mu, \nu=x, y, z$. The terms $\sigma_{\mu \nu}^{d}$ and $\sigma_{\mu \nu}^{\text {nd }}$ stem from the diagonal and nondiagonal part of the density operator $\hat{\rho}$, respectively, in a given basis and $\left\langle J_{\mu}\right\rangle=\operatorname{Tr}\left(\hat{\rho} J_{\mu}\right)=\sigma_{\mu \nu} E_{\nu}$. In general, we have $\sigma_{\mu \nu}^{d}$ $=\sigma_{\mu \nu}^{d i f}+\sigma_{\mu \nu}^{c o l}$. The term $\sigma_{\mu \nu}^{d i f}$ describes the diffusive motion of electrons and the term $\sigma_{\mu \nu}^{c o l}$ the collision contributions or hopping. The former is given by

$$
\sigma_{\mu \nu}^{d i f}=\frac{\beta e^{2}}{S_{0}} \sum_{\zeta} f\left(E_{s}^{\sigma}\right)\left[1-f\left(E_{s}^{\sigma}\right)\right] \tau^{\zeta}\left(E_{s}^{\sigma}\right) v_{\mu}^{\zeta} v_{\nu}^{\zeta},
$$

where $\zeta \equiv\left(s, \sigma, k_{y}\right)$ denotes the quantum numbers, $v_{\mu}^{\zeta}$ $=\left\langle\zeta\left|v_{\mu}\right| \zeta\right\rangle$ is the diagonal element of the velocity operator $v_{\mu}$, and $f(\varepsilon)$ the Fermi-Dirac function. Further, $\tau^{\zeta}\left(E_{s}^{\sigma}\right)$ is the relaxation time for elastic scattering, $\beta=1 / k_{B} T$, and $S_{0}$ is the area of the system.

The term $\sigma_{\mu \nu}^{c o l}$ can be written in the form

$$
\begin{aligned}
\sigma_{y y}^{c o l}= & \frac{\beta e^{2}}{2 S_{0}} \sum_{\zeta, \zeta^{\prime}} \int_{-\infty}^{\infty} d \varepsilon \int_{-\infty}^{\infty} d \varepsilon^{\prime} \delta\left[\varepsilon-E_{s}^{\sigma}\left(k_{x}\right)\right] \delta\left[\varepsilon^{\prime}-E_{s^{\prime}}^{\sigma^{\prime}}\left(k_{x}^{\prime}\right)\right] f(\varepsilon) \\
& \times\left[1-f\left(\varepsilon^{\prime}\right)\right] W_{\zeta \zeta^{\prime}}\left(\varepsilon, \varepsilon^{\prime}\right)\left(y_{\zeta}-y_{\zeta^{\prime}}\right)^{2}
\end{aligned}
$$

where $y_{\zeta}=\langle\zeta|y| \zeta\rangle ; W_{\zeta \zeta^{\prime}}\left(\varepsilon, \varepsilon^{\prime}\right)$ is the transition rate. For elastic scattering by dilute impurities, of density $N_{I}$, we have

$$
W_{\zeta \zeta^{\prime}}\left(\varepsilon, \varepsilon^{\prime}\right)=\frac{2 \pi N_{I}}{\hbar S_{0}} \sum_{\mathbf{q}}|U(\mathbf{q})|^{2}\left|F_{\zeta \zeta^{\prime}}(u)\right|^{2} \delta\left(\varepsilon-\varepsilon^{\prime}\right) \delta_{k_{x}, k_{x}^{\prime}-q_{x}},
$$

where $u=l_{c}^{2} q^{2} / 2$ and $q^{2}=q_{x}^{2}+q_{y}^{2} . U(\mathbf{q})=\left(e^{2} / 2 \epsilon_{0} \epsilon\right) /\left(q+k_{s}\right)$ is the Fourier transform of the screened impurity potential with $\epsilon$ the static dielectric constant, $\epsilon_{0}$ the dielectric permittivity, and $k_{s}$ the screening wave vector.

The diffusion contribution given by Eq. (17) becomes

$$
\begin{aligned}
\sigma_{y y}^{d i f}= & \frac{e^{2}}{h} \frac{4 \beta u^{2} \tau}{\pi K} \sum_{s, \sigma} \int_{0}^{a / 2 l_{c}^{2}} d k_{y}\left(\Delta_{s}^{\sigma}\right)^{2} \sin ^{2}\left(K l_{c}^{2} k_{y}\right) f\left(E_{n, k_{y}}^{\sigma}\right)[1 \\
& \left.-f\left(E_{n, k_{y}}^{\sigma}\right)\right]
\end{aligned}
$$

with $\Delta_{s}^{\sigma}=\Delta_{s}^{ \pm}$given by Eq. (11). The related contribution $\sigma_{x x}^{d i f}$ is zero since the velocity $v_{x}$ vanishes.

For weak potential modulations we can neglect Landaulevel mixing, i.e., we can take $s^{\prime}=s$. Then noting that $\sigma_{x x}^{c o l}$ $=\sigma_{y y}^{c o l}, \quad \Sigma_{\mathbf{q}}=\left(S_{0} / 2 \pi\right) \int_{0}^{\infty} q d q=\left(S_{0} / 2 \pi l_{c}^{2}\right) \int_{0}^{\infty} d u, \quad$ and $\quad \Sigma_{k_{x}}$ $=\left(S_{0} / 2 \pi l_{c}^{2}\right)$, the collisional contribution given by Eq. (18) takes the form

$$
\begin{aligned}
\sigma_{y y}^{c o l}= & \frac{e^{2}}{\hbar} \frac{N_{I} \beta}{2 A_{0}} \sum_{s, \sigma, k_{y}} \int_{0}^{\infty} d u\left|F_{s s}^{\sigma}(u)\right|^{2} u \int_{-\infty}^{\infty} d \varepsilon\left[\delta\left(\varepsilon-E_{s}^{\sigma}\right)\right]^{2} f(\varepsilon)[1 \\
& -f(\varepsilon)]\left|U\left(\sqrt{2 u / l_{c}^{2}}\right)\right|^{2}
\end{aligned}
$$

where

$$
\begin{aligned}
& \left|F_{s s}^{-}(u)\right|^{2}=\left\{L_{s-1}(u)+\mathcal{D}_{s}^{2} L_{s}(u)\right\}^{2} e^{-u} / \mathcal{A}_{s}^{2}, \\
& \left|F_{s s}^{+}(u)\right|^{2}=\left\{\mathcal{D}_{s}^{2} L_{s-1}(u)+L_{s}(u)\right\}^{2} e^{-u} / \mathcal{A}_{s}^{2} .
\end{aligned}
$$

The exponential $e^{-u}$ favors small values of $u$. Assuming $b=k_{s}^{2} l_{c}^{2} / 2 \gg u$ we may neglect the term $2 u / l_{c}^{2}$ in the expression for $U\left(\sqrt{2 u / l_{c}^{2}}\right)$ and define $U_{0}=U(0)$. We then obtain

$$
\sigma_{y y}^{c o l}=\frac{e^{2}}{h} \frac{N_{I} U_{0}^{2} \beta}{\pi a \Gamma} \sum_{s, \sigma} \int_{0}^{a / 2 l_{c}^{2}} d k_{y} I_{s}^{\sigma} f(\varepsilon)[1-f(\varepsilon)]
$$

where

$$
I_{s}^{ \pm}=\left[(2 s \pm 1) \mathcal{D}_{s}^{4}-2 s \mathcal{D}_{s}^{2}+2 s \pm 1\right] / \mathcal{A}_{s}^{2} .
$$

The impurity density $N_{I}$ determines the Landau Level broadening $\Gamma=W_{\zeta \zeta^{\prime}}\left(\varepsilon, \varepsilon^{\prime}\right) / \hbar$. Evaluating $W_{\zeta \zeta^{\prime}}\left(\varepsilon, \varepsilon^{\prime}\right) / \hbar$ in the $u \rightarrow 0$ limit without taking into account the SOI, we obtain $N_{I} \approx 4 \pi\left[\left(2 \epsilon \epsilon_{0} / e^{2}\right)\right]^{2} \Gamma / \hbar$.

The Hall conductivity $\sigma_{x y}^{\text {nd }}$ is given by 

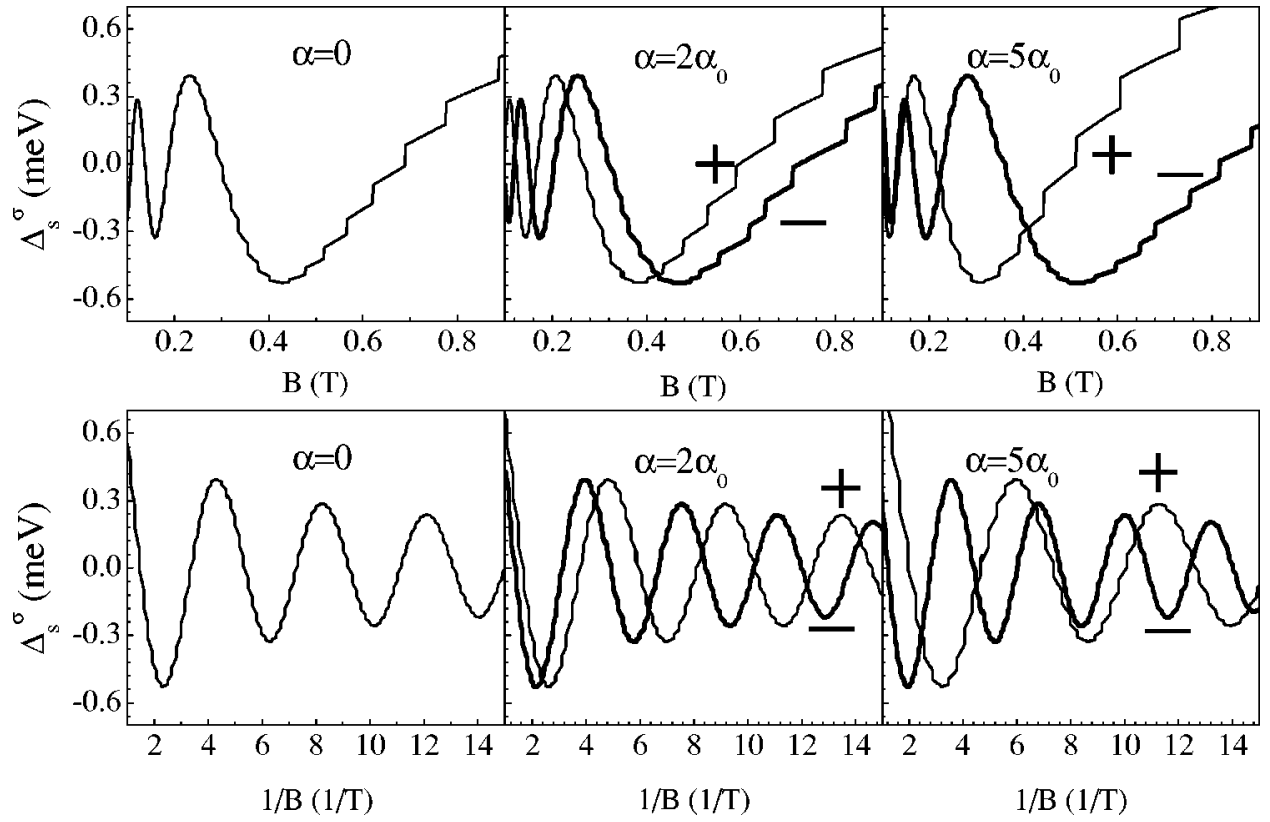

FIG. 1. The quantity $\Delta_{s}^{ \pm}$of Eq. (11) vs magnetic field $B$ (upper panels) and vs inverse magnetic field $1 / B$ (lower panels), at the Fermi level, for different values of the strength $\alpha$. The modulation period is $a=3500 \AA$ and the modulation strength $V_{0}=0.5 \mathrm{meV}$.

$$
\begin{aligned}
\sigma_{x y}^{n d}= & \frac{2 i \hbar e^{2}}{S_{0}} \sum_{\zeta, \zeta^{\prime}} f\left(E_{\zeta}\right)\left[1-f\left(E_{\zeta}^{\prime}\right)\right]\left\langle\zeta\left|v_{x}\right| \zeta^{\prime}\right\rangle \\
& \times\left\langle\zeta^{\prime}\left|v_{y}\right| \zeta\right\rangle \frac{1-e^{\beta\left(E_{\zeta}-E_{\zeta}^{\prime}\right)}}{\left(E_{\zeta}-E_{\zeta}^{\prime}\right)^{2}}, \quad \zeta^{\prime} \neq \zeta .
\end{aligned}
$$

The resistivity tensor $\rho_{\mu \nu}$ is given in terms of the conductivity tensor $\sigma_{\mu \nu}$ upon using the standard expressions $\rho_{x x}$ $=\sigma_{y y} / S, \quad \rho_{y y}=\sigma_{x x} / S, \quad \rho_{y x}=\rho_{x y}=-\sigma_{y x} / S$, where $S=\sigma_{x x} \sigma_{y y}$ $-\sigma_{x y} \sigma_{y x}$.

\section{NUMERICAL RESULTS}

In this section we present numerical results for the bandwidth and the two conductivities given by Eqs. (20) and (23) for various values of the SOI strength $\alpha$, of the modulation strength $V_{0}$ and period $a$, of the electron density $n_{e}$, and of the temperature $T$. We measure $\alpha$ in units of $\alpha_{0}$ $=10^{-11} \mathrm{eV} \mathrm{m}, n_{e}$ in units of $n_{0}=10^{-11} / \mathrm{cm}^{2}$, and use the effective mass of InAs $m^{*}=0.05 m_{0}$ with $m_{0}$ the free-electron mass.

In Fig. 1 we plot $\Delta_{s}^{ \pm}$, given by Eq. (11) and directly related to the bandwidth $2\left|\Delta_{s}^{ \pm}\right|$, at the Fermi level, as a function of the magnetic field $B$ in the upper panels and as a function of the inverse magnetic field $1 / B$ in the lower panels. The other parameters are $a=3500 \AA, T=2 \mathrm{~K}, n_{e}=3 n_{0}$, and $V_{0}$ $=0.5 \mathrm{meV}$. We plot $\Delta_{s}^{ \pm}$and not $2\left|\Delta_{s}^{ \pm}\right|$so that the oscillations are seen more clearly. Comparing the $\alpha=0$ panel with the $\alpha \neq 0$ ones, we see clearly, for $\alpha \neq 0$, the contributions from the + and - branches. The large-amplitude oscillations, for low $B$ in the upper panels and for high $1 / B$ in the lower panels, are the Weiss oscillations whereas the step-like behavior on the right side of the upper panels is due to the small-amplitude $\mathrm{SdH}$ ones. On the scale used the latter are barely visible on the very left side in the lower panels. The phase shift between the oscillations of $\Delta_{s}^{+}$and $\Delta_{s}^{-}$and their slightly different frequencies described by Eq. (16) lead to the beating patterns of the conductivities shown below. For example, for $\alpha=2 \alpha_{0}$ the oscillations of the bandwidth $2\left|\Delta_{s}^{ \pm}\right|$, given after Eq. (16), have a period $\Omega^{+}=2.14 \mathrm{~T}^{-1}$ in the + branch and a period $\Omega^{-}=1.76 \mathrm{~T}^{-1}$ in the - branch.

In Fig. 2 we plot the conductivities versus the inverse of the magnetic field $B$ for different values of $\alpha$ and a shorter modulation period $a=800 \AA$. The upper curve is the collisional conductivity, given by Eq. (23), and the lower one the diffusive conductivity, given by Eq. (20). Notice the absence of a beating pattern for $\alpha=0$ and its development for $\alpha \neq 0$. For finite $\alpha$, the longer-period beating pattern of the Weiss oscillations is observed in the diffusive curves and the

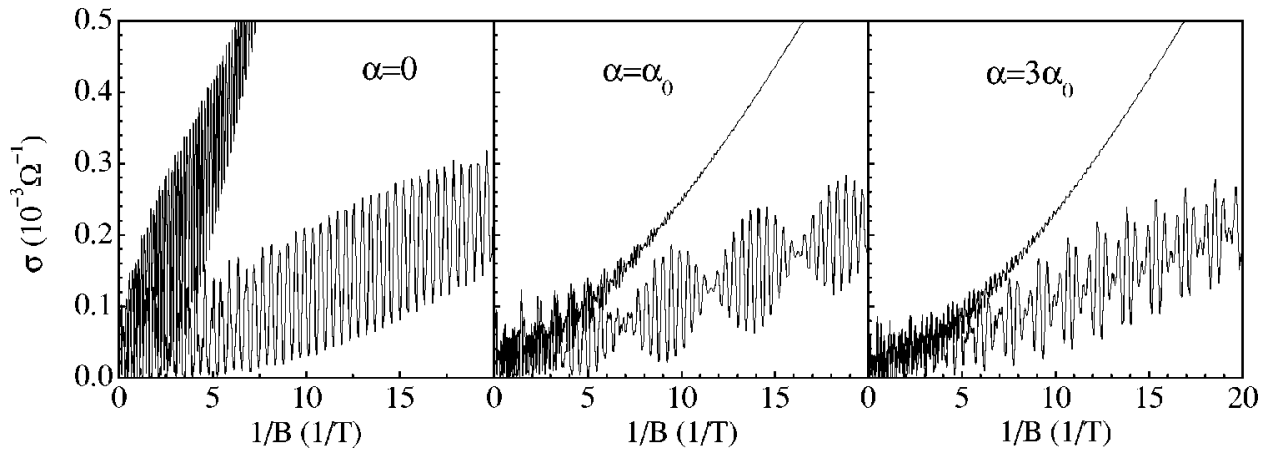

FIG. 2. Conductivities vs inverse magnetic field $B$ for different values of $\alpha$ with $a=800 \AA, T$ $=1 \mathrm{~K}, n_{e}=3 n_{0}$, and $V_{0}=0.3 \mathrm{meV}$. The upper (lower) curves show the collisional (diffusive) contribution. 


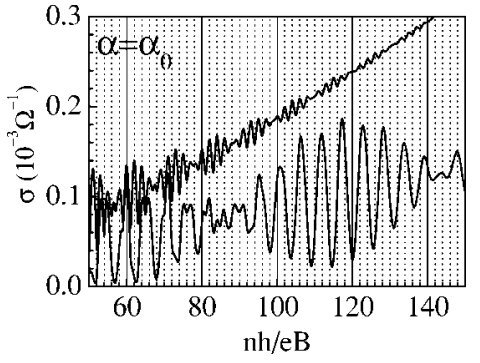

FIG. 3. Conductivities vs filling factor $n h / e B$ for the panel $\alpha$ $=\alpha_{0}$ of Fig. 2. The dashed vertical lines show the even filling factor values and the curves are marked as in Fig. 2.

shorter-period beating pattern of the $\mathrm{SdH}$ oscillations ${ }^{17}$ in the collisional curves. The reason is that at low magnetic fields and low temperatures the Weiss oscillations dominate the diffusive conductivity while the $\mathrm{SdH}$ oscillations dominate the collisional conductivity. In the former the energy correction due to the modulation, given by Eqs. (9) and (10), enters mainly the square of $v_{y}^{ \pm}$and the argument of the Fermi function, cf. Eqs. (17) and (20), whereas in the latter it enters essentially only through the argument of the Fermi function, cf. Eqs. (18) and (24).

To see the oscillations shown in Fig. 2 more clearly, we plot the conductivities versus filling factor $n h / e B$ in Fig. 3 for $\alpha=\alpha_{0}$ and $n_{e}=3 n_{0}$. As can be seen, the collisional conductivity (upper curve) shows a beating pattern of the $\mathrm{SdH}$ oscillations resulting from the different Landau-level separations in the + and - spin branches. The index $s^{ \pm}$at the Fermi energy is expressed approximately as $s^{ \pm}$ $=\left(\pi n_{e} \hbar \mp m^{*} \alpha \sqrt{2 \pi n_{e}} / \hbar\right) / e B$. The resulting period of the beating pattern, measured in units of inverse magnetic field, is $2 \hbar k_{\alpha} k_{F} / e$ or $0.85 \mathrm{~T}^{-1}$ in Fig. 3. We notice that a transition from conductivity maxima at even filling factors to conductivity maxima at odd filling factors occurs between adjacent wraps of the $\mathrm{SdH}$ oscillations. This can be understood by checking the DOS of the system. As shown in Fig. 4, when the subband broadening is comparable to the subband separation, a beating pattern appears in the DOS, with SOI present and modulation absent, and each DOS peak corresponds to one pair of spin levels. Because the spin-up and spin-down levels have different separations, there is one unpaired spin level at each node of the beating pattern. As a result, in one wrap of the DOS oscillations there is an even number of levels below each pair and the DOS has a peak at odd filling factors, while in the next wrap there is an odd number of levels below each pair and the DOS has a peak at even filling factors. When the Fermi energy passes through

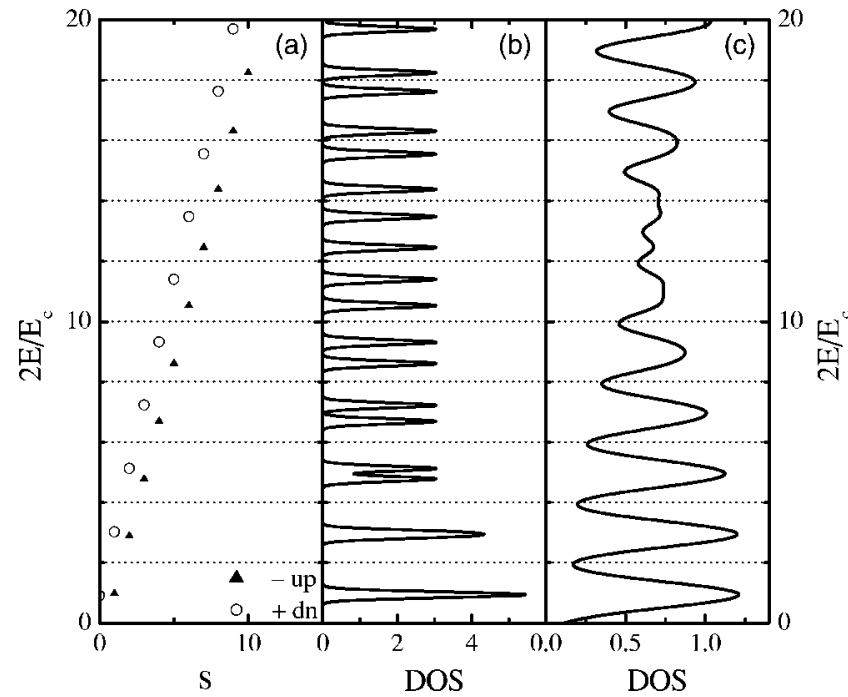

FIG. 4. (a) Subband energy vs index $s$ in the absence of modulation. The DOS vs energy is shown in (b) for subband broadening $\Gamma=0.1 \mathrm{meV}$ and in (c) for $\Gamma=0.5 \mathrm{meV}$. When $E$ is the Fermi energy the quantity $2 E / E_{c}$ with $E_{c}=\hbar \omega_{c}$ is approximately the filling factor.

the \pm branches and the DOS is as described above, the collisional conductivity shows a beating pattern with an evenodd filling factor transition. Although here the modulation is present, it is very weak and leaves the oscillations of the collisional conductivity nearly intact. A complementary way of seeing how the beating pattern is formed, is to plot separately $\sigma^{c o l,-}$ and $\sigma^{c o l,+}$. Both contributions oscillate with slightly different frequencies and their sum shows the beating pattern of Fig. 3. The period of this pattern, in units of inverse of magnetic field, is $e a / 4 \hbar k_{\alpha}$ or $4.63 \mathrm{~T}^{-1}$ in Fig. 3. A similar even-odd filling factor transition was also observed for strong modulations, which make the Landau levels overlap, in the absence of SOI and was explained by the behavior of the corresponding DOS. ${ }^{20}$ The diffusive conductivity (lower curve) shows mainly a beating pattern of the Weiss oscillations since here the SdH oscillations are very weak.

Above we observed a beating pattern in the $\mathrm{SdH}$ and Weiss oscillations occurring, respectively, in the collisional and diffusive conductivities, versus filling factor when varying the magnetic field at a fixed electron density. If we vary the electron density and fix the magnetic field $B$, the beating pattern of the SdH oscillations holds because it corresponds to the Fermi energy passing through the DOS with beating pattern. However, we do not observe a beating pattern in the Weiss oscillations. This can be explained by Eq. (16), from where we see that, for fixed $B$, the bandwidths of the two

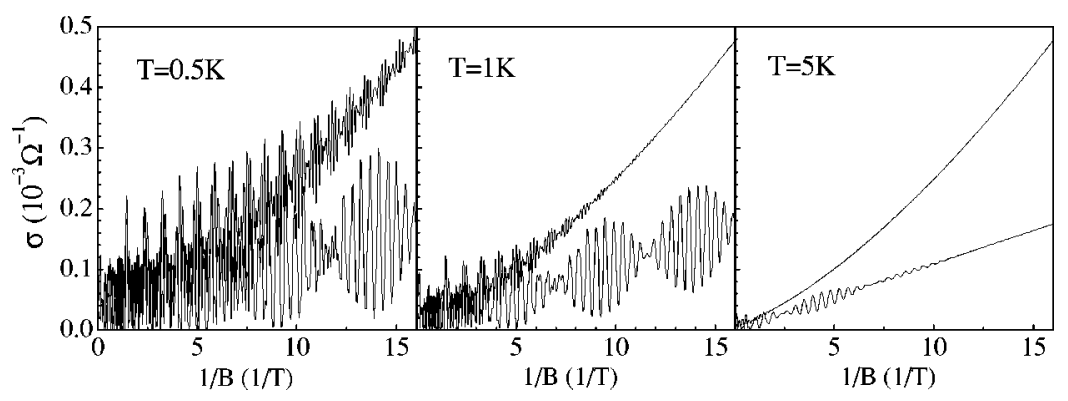

FIG. 5. Conductivities vs inverse magnetic field $B$ for different temperatures with $\alpha=\alpha_{0}$ and $n_{e}=3 n_{0}$. The upper (lower) curves show the collisional (diffusive) contribution. 

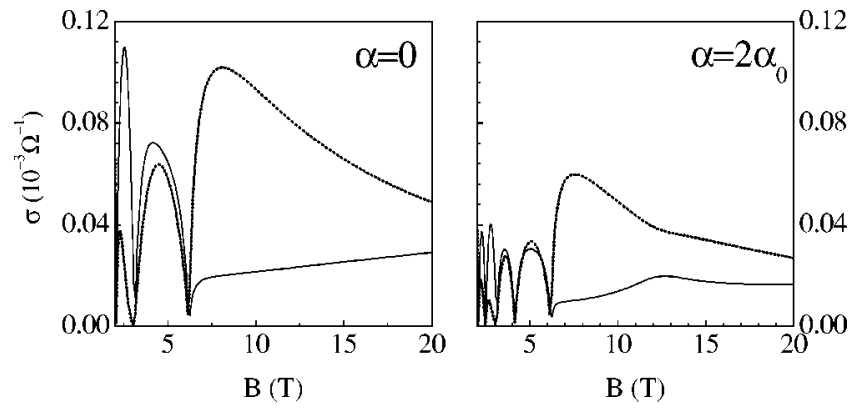

FIG. 6. Conductivities vs magnetic field $B$ for two different values of $\alpha$. The temperature is $T=1 \mathrm{~K}$ and the density $n_{e}=3 n_{0}$. The dotted (solid) curves show the collisional (diffusive) conductivity.

series of spin levels oscillate with the same frequency as a function of the electron density $n_{e}$ though with different phases. For a system without the potential modulation, the diffusive conductivity disappears and we observe only a beating pattern of the $\mathrm{SdH}$ oscillations in the collisional conductivity.

In Fig. 5 we plot again the conductivities versus the inverse of the magnetic field $B$ for different values of the temperature, $\alpha=\alpha_{0}, n_{e}=3 n_{0}$, and $V_{0}=0.3 \mathrm{meV}$. The two curves are marked as in Fig. 2. Notice that beating pattern exists for all temperatures but the oscillation amplitude decreases with increasing temperature and nearly disappears at $T \approx 5 \mathrm{~K}$ for the density and SOI strength used.

In Fig. 6 we plot the conductivities vs magnetic field $B$, for rather strong values of $B$, and different $\alpha$. The temperature is $T=1 \mathrm{~K}$. The dotted (solid) curves show the collisional (diffusive) conductivity. The SOI splits each Landau subband and reduces the DOS inside it. As a result, a reduction in the oscillation amplitude and a splitting of the $(\mathrm{SdH})$ oscillations are observed in the $\alpha=2 \alpha_{0}$ panel compared with the $\alpha=0$ one. For the high magnetic fields involved here, the period of the Weiss oscillations is very long and both the diffusive and collisional conductivity curves show the $\mathrm{SdH}$ oscillations with the same phase.

In Fig. 7 we plot the conductivities vs magnetic field $B$ for different densities, $\alpha=\alpha_{0}$, and temperature $T=1 \mathrm{~K}$. Again the two curves are marked as in Fig. 2. Notice how increasing the density and thus changing the position of the Fermi level relative to those of the + and - branches closest to it modifies the beating pattern.

In Fig. 8 we plot the conductivities vs magnetic field $B$ for different $\alpha$. The density is $n_{e}=3 n_{0}$ and the temperature $T$

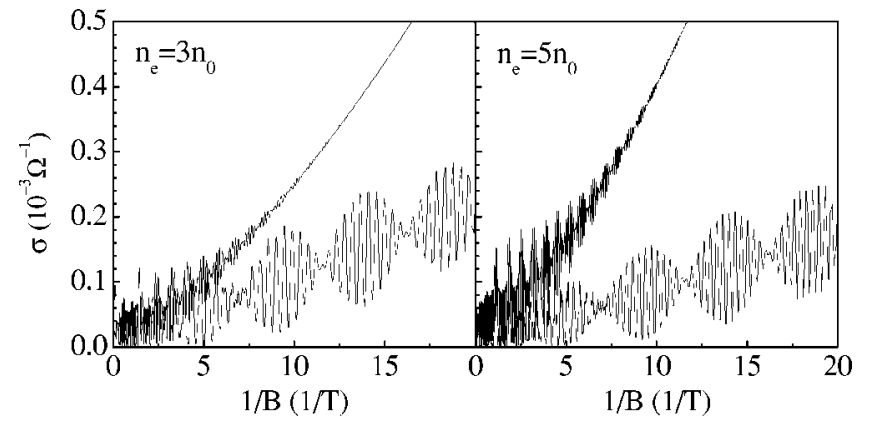

FIG. 7. Conductivities vs inverse magnetic field $B$ for different densities, $\alpha=\alpha_{0}$, and temperature $T=1 \mathrm{~K}$. The upper (lower) curves show the collisional (diffusive) conductivity.

$=2 \mathrm{~K}$. The upper (lower) curves are the collisional (diffusive) contributions. The diffusive curve shows mainly the Weiss oscillations at low $B$ and at high $B$ the short-period $\mathrm{SdH}$ oscillations in addition to the long-period Weiss oscillations. The collisional curve shows clearly the $\mathrm{SdH}$ oscillations for $\alpha=0$ and a beating pattern of the SdH oscillation for finite $\alpha$.

We now address the issue of the Hall conductivity $\sigma_{x y}^{n d}$. In the absence of modulation and presence of SOI, it has been evaluated in Ref. 17 for rather strong fields $B \geqslant 1 \mathrm{~T}$ and shows two series of quantum Hall plateaus, for strong $\alpha$ ( $\alpha$ $\approx 10 \alpha_{0}$ ), corresponding to the two branches developed due the SOI. The 1D modulation removes the $k_{y}$ degeneracy of the Landau levels $E_{s}$ and broadens them into bands with eigenvalues $E_{s, k_{y}}$. From Eq. (26) we see that this may affect the Hall conductivity at weak magnetic fields when the broadening $\Delta_{s}$ is comparable to the energy $\hbar \omega_{c}$. In the presence of modulation and absence of SOI, it has been evaluated in Ref. 3 for weak fields $B \leqslant 1 \mathrm{~T}$ and shows very smallamplitude oscillations expressed mainly through the energy difference between the $n$ and $n \pm 1$ Landau levels. Here the interest is in the region of weak fields $B \leqslant 1 \mathrm{~T}$ for which the Weiss oscillations appear. Despite the fact that $\Delta_{s}$ is comparable to $\hbar \omega_{c}$, it exhibits again very small-amplitude oscillations so far not observed for weak modulations. ${ }^{21}$ If we neglect these oscillations, it is approximately given by $\sigma_{x y}^{\text {nd }}$ $\approx n e / B$.

Experimentally one usually measures the resistivity $\rho_{\mu \nu}$. Using the expressions given at the end of Sec. III for $\rho_{\mu \nu}$, $\sigma_{x y}^{n d} \approx n e / B$, and the results for $\sigma_{y y}$ and $\sigma_{x x}$, we show in Fig. 9 the resistivities divided by the magnetic field $\rho_{x x} / B$, for

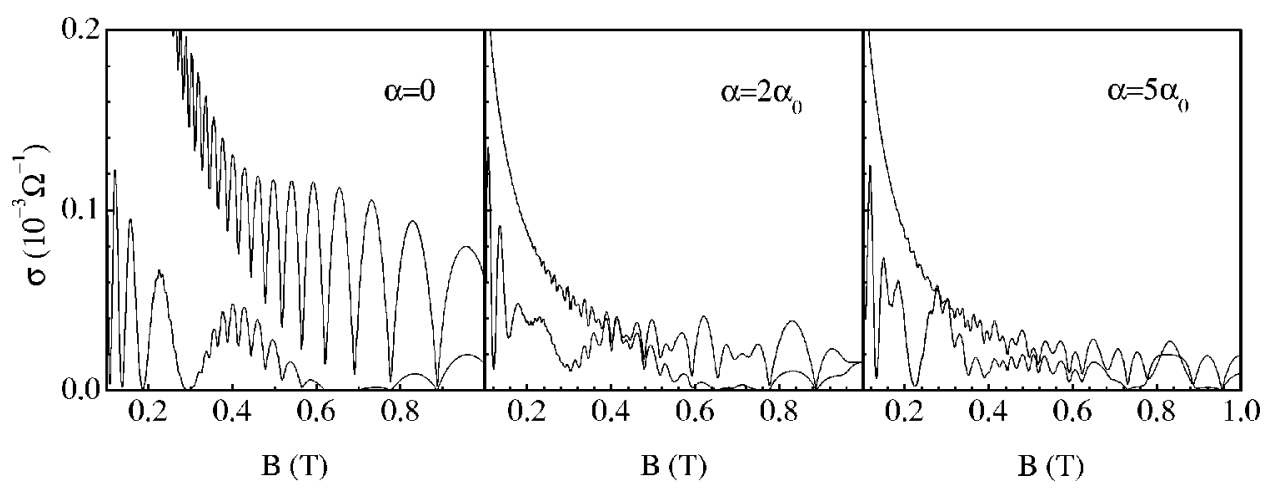

FIG. 8. Conductivities vs magnetic field $B$ for different $\alpha$. The density is $n_{e}=3 n_{0}$, the modulation period $a=3500 \AA$, and the temperature $T=2 \mathrm{~K}$. The upper (lower) curves show the collisional (diffusive) conductivity. 


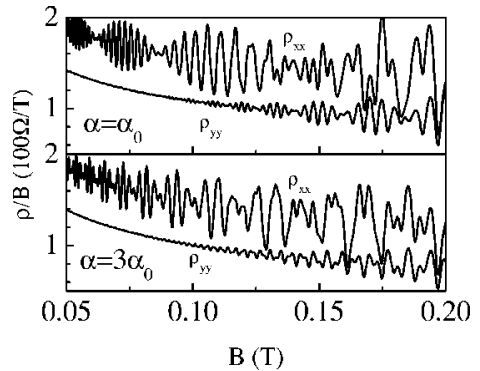

FIG. 9. Resistivities divided by the field $B, \rho_{x x} / B$ and $\rho_{y y} / B$, vs field $B$ for $\alpha=\alpha_{0}$ (upper panel) and $\alpha=3 \alpha_{0}$ (lower panel). The other parameters are the same as in Fig. 2.

$\alpha=\alpha_{0}$ (upper panel) and $\alpha=3 \alpha_{0}$ (lower panel), as a function of the magnetic field for a system with $n_{e}=3 n_{0}$ and otherwise the same parameters as in Fig. 7. For $\alpha=\alpha_{0}$ and in the lowfield region, in which the $\mathrm{SdH}$ oscillations are absent, a beating pattern of the Weiss oscillations is clearly observed in the $\rho_{x x}$ curve. The $\rho_{y y}$ curve exhibits a beating pattern only for the $\mathrm{SdH}$ oscillations since they result only from collisional current contributions and the Weiss oscillations are very weak as the diffusive contributions to $\rho_{y y} \propto \sigma_{x x}$ vanish. For $\alpha=3 \alpha_{0}$ though the beating patterns change: that of the Weiss oscillations, when discernible in $\rho_{x x}$, becomes shorter and that of the $\mathrm{SdH}$ oscillations in $\rho_{y y}$ disappears. For completeness it should be mentioned, though not shown, that for $\alpha$ $=0$ there are no beating patterns in either the Weiss ${ }^{3}$ or $\mathrm{SdH}$ (Ref. 17) oscillations.

\section{CONCLUDING REMARKS}

We evaluated quantum mechanically the dc conductivities of a 2DEG in the presence of SOI of strength $\alpha$, of a normal magnetic field $B$, and of a weak 1D potential modulation of strength $V_{0}$ and of period $a$. The SOI splits the Landau levels, for $\alpha=0$, in two unequally spaced energy branches. As in the absence of SOI, the modulation broadens the levels of these branches into bands and their bandwidths oscillate independently with the field $B$. This gives rise to two flat-band conditions, instead of one for $\alpha=0$, and to the beating patterns of the Weiss oscillations. As for the SdH oscillations, their beating patterns for weak $\alpha$ are nearly independent of the modulation, at least as long as the latter is weak, and agree with those of Ref. 17 obtained in the absence of modulation. However, for strong $\alpha$ an additional structure is ob- tained and the $\mathrm{SdH}$ oscillations split in two, cf. Fig. 6. We also noticed the even-odd filling factor transition in the $\mathrm{SdH}$ oscillations and explained it with the help of the broadened DOS. A similar observation was made in Ref. 20 for strong modulations and was explained by the corresponding DOS.

Regarding the Weiss oscillations the results for the diffusive conductivity agree, as expected, for the relevant weak magnetic fields and high quantum numbers $s$, with those of the classical evaluation of Ref. 18. However, the results for the collisional conductivity could not be obtained by a classical treatment and, to our knowlege, are new. It is well known that this collisional or hopping conductivity describes the SdH oscillations which cannot be treated classically. This explains their absence from Ref. 18 and their modification for strong $\alpha$ as well as for strong $B$, cf. Fig. 6, presented here.

For weak $\alpha$ both conductivities exhibit beating patterns. Those of the diffusive conductivity pertain to the Weiss oscillations and are due to the two independent frequencies involved in the bandwidths of the + and - branches created by the SOI whereas those of the collisional conductivity pertain to the $\mathrm{SdH}$ oscillations and have a similar explanation though the two frequencies involved here are not those of the bandwidths, see the discussion of Fig. 3. As we saw though, these patterns weaken or disappear rather quickly upon increasing the temperature or the strength $\alpha$. On the electron density $n_{e}$ though, they appear to have a rather weak dependence, cf. Fig. 7, at least as long as $n_{e}$ falls in the range of the usual experimental densities of a 2DEG.

We are not aware of any directly relevant experimental work. We hope though that the findings described above will motivate experiments in which the magnetoresistivities along the $x$ and $y$ directions could be measured in a weakly modulated 2DEG in the presence of SOI. For a 1D modulation along the $x$ direction, the diffusive and collisional contributions to the conductivity can be obtained separately using the relations $\sigma_{y y}=\sigma_{y y}^{d i f}+\sigma_{y y}^{c o l}$ and $\sigma_{x x}=\sigma_{x x}^{c o l}$. Combining them with the standard relations given after Eq. (26), gives the magnetoresistivities.

\section{ACKNOWLEDGMENTS}

This work was supported by the Canadian NSERC Grant No. OGP0121756, by the Flemish Science Foundation (FWO-VI), the Belgian Science Policy, and the EU-CERION project.

\footnotetext{
*Electronic address: xuefeng@alcor.concordia.ca

†Electronic address: takis@alcor.concordia.ca

†Electronic address: peeters@uia.ac.be

${ }^{1}$ D. Weiss, K. von Klitzing, K. Ploog, and G. Weimann, Europhys. Lett. 8, 179 (1989); R. W. Winkler, J. P. Kotthaus, and K. Ploog, Phys. Rev. Lett. 62, 1177 (1989).

${ }^{2}$ R. R. Gerhardts, D. Weiss, and K. v. Klitzing, Phys. Rev. Lett. 62, 1173 (1989); C. W. J. Beenakker, ibid. 62, 2020 (1989); P.
}

Vasilopoulos and F. M. Peeters, ibid. 63, 2120 (1989); P. Streda and A. H. MacDonald, Phys. Rev. B 41, 11892 (1990); F. M. Peeters and P. Vasilopoulos, ibid. 42, 5899 (1990).

${ }^{3}$ F. M. Peeters and P. Vasilopoulos, Phys. Rev. B 46, 4667 (1992).

${ }^{4}$ S. Datta and B. Das, Appl. Phys. Lett. 56, 665 (1990); X. F. Wang, P. Vasilopoulos, and F. M. Peeters, Phys. Rev. B 65, 165217 (2002).

${ }^{5}$ D. D. Awschalom and J. M. Kikkawa, Phys. Today 52, 33 (1999). 
${ }^{6}$ E. Tutuc, E. P. De Poortere, S. J. Papadakis, and M. Shayegan, Phys. Rev. Lett. 86, 2858 (2001).

${ }^{7}$ J. P. Lu, J. B. Yau, S. P. Shukla, M. Shayegan, L. Wissinger, U. Rossler, and R. Winkler, Phys. Rev. Lett. 81, 1282 (1998).

${ }^{8}$ A. G. Aronov and Y. B. Lyanda-Geller, Phys. Rev. Lett. 70, 343 (1993); A. F. Morpurgo, J. P. Heida, T. M. Klapwijk, B. J. van Wees, and G. Borghs, ibid. 80, 1050 (1998); B. Molnar, F. M. Peeters, and P. Vasilopoulos, Phys. Rev. B 69, 155335 (2004).

${ }^{9}$ S. D. Ganichev, E. L. Ivchenko, V. V. Bel'kov, S. A. Tarasenko, M. Sollinger, D. Weiss, W. Wegscheider, and W. Prettl, Nature (London) 417, 153 (2002).

${ }^{10}$ F. Mireles and G. Kirczenow, Phys. Rev. B 66, 214415 (2002).

${ }^{11}$ J. Sinova, D. Culcer, Q. Niu, N. A. Sinitsyn, T. Jungwirth, and A. H. MacDonald, Phys. Rev. Lett. 92, 126603 (2004).

${ }^{12}$ G. Dresselhaus, Phys. Rev. 100, 580 (1955); E. A. de Andrada e silva, G. C. La Rocca, and F. Bassani, Phys. Rev. B 50, 8523 (1994).

${ }^{13}$ E. I. Rashba, Sov. Phys. Solid State 2, 1109 (1960).
${ }^{14}$ H. L. Stormer, Z. Schlesinger, A. Chang, D. C. Tsui, A. C. Gossard, and W. Wiegmann, Phys. Rev. Lett. 51, 126 (1983).

${ }^{15}$ J. Luo, H. Munekata, F. F. Fang, and P. J. Stiles, Phys. Rev. B 38, 10142 (1988); B. Das, D. C. Miller, S. Datta, R. Reifenberger, W. P. Hong, P. K. Bhattacharya, J. Singh, and M. Jaffe, ibid. 39, 1411 (1989).

${ }^{16}$ Y. A. Bychkov and E. I. Rashba, J. Phys. C 17, 6039 (1984).

${ }^{17}$ X. F. Wang and P. Vasilopoulos, Phys. Rev. B 67, 085313 (2003).

${ }^{18}$ L. Magaril, Superlattices Microstruct. 16, 257 (1994).

${ }^{19}$ P. Vasilopoulos and C. M. Van Vliet, J. Math. Phys. 25, 1391 (1984).

${ }^{20}$ J. Shi, F. M. Peeters, K. W. Edmonds, and B. L. Gallagher, Phys. Rev. B 66, 035328 (2002).

${ }^{21}$ For strong 1D modulations oscillations in $\sigma_{x y}^{n d}$ have been observed by K. W. Edmonds, B. L. Gallagher, P. C. Main, N. Overend, R. Wirtz, A. Nogaret, M. Henini, C. H. Marrows, B. J. Hickey, and S. Thoms, Phys. Rev. B 64, 041303(R) (2001). 